\title{
Fast Algorithms for the Solution of Eigenfunction Problems for One-Dimensional Self-Adjoint Linear Differential Operators
}

\author{
Mark Tygert* \\ Research Report YALEU/DCS/RR-1339 \\ December 7, 2005
}

\begin{abstract}
We cite techniques that immediately yield theoretically efficient algorithms for computing solutions to eigenfunction problems for self-adjoint linear differential operators of any finite order acting on real-valued functions on the interval $[-1,1]$; these algorithms incur computational costs that are nearly optimally small, to within factors that are constant multiples of small powers of the logarithm of the problem size. However, the factors in the computational costs are in general too large for practical applications; the algorithms would appear to require careful, detailed optimizations for each particular application in order to be useful in practice.
\end{abstract}

For any positive real number $\varepsilon$, positive integer $m$, and matrix $A$, we say that $A$ is semiseparable to (absolute) precision $\varepsilon$, with a semiseparability rank of at most $m$, to mean that any contiguous rectangular block of $A$ that does not contain any entry from the diagonal of $A$ has at most $m$ singular values greater than $\varepsilon$.

Observation 1 The adaptive versions of the discretizations described in [2], [5], and [7] recast eigenfunction problems for self-adjoint linear differential operators of any finite order acting on real-valued functions on the interval $[-1,1]$ as eigenvector problems for self-adjoint semiseparable matrices (provided that all boundary conditions are linear whenever any boundary conditions are prescribed).

Observation 2 For any positive real number $\varepsilon$, positive integers $m$ and $N$, and $N \times N$ matrix $A$ that is self-adjoint, has $N$ distinct eigenvalues, and is semiseparable to precision $\varepsilon$, with a semiseparability rank of at most $m$, the techniques introduced in [4], [3], and [1] immediately yield well-conditioned algorithms for computing

1. the $N$ eigenvalues of $A$, at a $\operatorname{cost}$ of $\mathcal{O}(m \cdot N \cdot \ln N)$ operations and memory storage elements,

2. the $N$ entries of any of the eigenvectors of $A$, at a cost of $\mathcal{O}(m \cdot N)$,

3. the $N$ entries of any linear combination of the normalized eigenvectors of $A$ from the coefficients of the eigenvectors in the linear combination, at a cost of $\mathcal{O}(m \cdot N \cdot \ln N)$, and

4. the $N$ coefficients of the eigenvectors in any linear combination of the normalized eigenvectors of $A$ from the entries of the linear combination, at a cost of $\mathcal{O}(m \cdot N \cdot \ln N)$;

* Partially supported by the U.S. DoD under a 2001 NDSEG Fellowship. 
these costs pertain to computations performed to precision $\varepsilon$ and with $A$ itself stored efficiently, at a cost of $\mathcal{O}\left(m^{2} \cdot N \cdot \ln N\right)$.

As is implicit in [1], combining Observations 1 and 2 immediately yields theoretically efficient algorithms for computing solutions to eigenfunction problems for self-adjoint linear differential operators of any finite order; these algorithms incur computational costs that are nearly optimally small, to within factors that are constant multiples of small powers of the logarithm of the problem size. However, the factors in the computational costs are in general too large for practical applications. Techniques such as those described in [6] reduce the factors for specific applications.

\section{Acknowledgements}

We would like to thank Vladimir Rokhlin for many influential, pertinent discussions and Mauro Maggioni, Michael O'Neil, and Yoel Shkolnisky for proofreading.

\section{References}

[1] S. Chandrasekaran And M. Gu, A divide-and-conquer algorithm for the eigendecomposition of symmetric block-diagonal plus semiseparable matrices, Numer. Math., 96 (2004), pp. 723-731.

[2] L. Greengard And V. Rokhlin, On the numerical solution of two-point boundary value problems, Comm. Pure Appl. Math., 44 (1991), pp. 419-452.

[3] M. Gu And S. C. Eisenstat, A stable and efficient algorithm for the rank-1 modification of the symmetric eigenproblem, SIAM J. Matrix Anal. Appl., 15 (1994), pp. 1266-1276.

[4] — A divide-and-conquer algorithm for the symmetric tridiagonal eigenproblem, SIAM J. Matrix Anal. Appl., 16 (1995), pp. 172-191.

[5] J.-Y. Lee And L. Greengard, A fast adaptive numerical method for stiff two-point boundary value problems, SIAM J. Sci. Comput., 18 (1997), pp. 403-429.

[6] V. Rokhlin And M. Tygert, Fast algorithms for spherical harmonic expansions, Tech. Rep. 1309, Yale University, Dept. of Computer Science, December 2004.

[7] P. Starr and V. Rokhlin, On the numerical solution of two-point boundary value problems II, Tech. Rep. 802, Yale University, Dept. of Computer Science, June 1990. 